\title{
Synthesis and Antiviral Evaluation of Novel Acyclic Nucleosides
}

\author{
Joon Hee Hong" and Ok Hyun Ko \\ College of Phamacy, Chosun liniversity, Gwangin 501-759, Korea \\ Recened Ma: 7,2003
}

\begin{abstract}
A very short and concise synthetic route for a novel acyclic version of $d 4 T$ is described. The required quaternary carbon was successfully installed using a [3,3]-sigmatropic rearrangement. The condensation of the mesylates 16-18 with an adenine base under standard nucleophilic substitution conditions $\left(\mathrm{K}_{2} \mathrm{CO}_{-}\right.$, $18-\mathrm{Cr}_{\text {rown- }}$ 6. DMF) in addition to deblocking afforded the target acyclic nucleosides 22-24. In addition, the antiviral evaluations against various viruses were performed.
\end{abstract}

Key Words : Acyclic nucleosides. [3,3]-Sigmatropic rearrangement, Antiviral agents

\section{Introduction}

The discovery of acyclovir ${ }^{1}$ as an antiherpes agent ignited the search for new antiviral nucleosides with a disconnected chain resulting from the omission of bonds from the pentose or cyclopentane rings. During the past twenty years, many new synthetic schemes for various acyclic nucleoside ${ }^{2}$ analogues have been reported and many of these molecules have exhibited promising antiviral activities." Among them. deciclovir, ${ }^{4}$ ganciclovir, ${ }^{5}$ penciclovir. famciclovir ${ }^{7}$ have exhibited potent antiviral activity against HBV. HIV, as well as the herpes virus. Furthermore, the recent approval of bis(POC)PMPA ${ }^{8}$ by the FDA as an anti-HIV agent warrants further searches for acyclic nucleosides as chemotherapeutic agents (Figure 1).

Nevertheless, the utility of these drugs is limited due to their toxicity and side effects, as well as the emergence of drug resistant viral strains. Therefore, it is essential to search

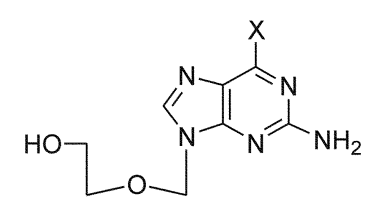

Acyclovir: $\mathrm{X}=\mathrm{OH}$ Deciclovir: $X=H$<smiles>Nc1nc2c(ncn2COC(CO)(CO)C(Cl)Cl)c(=O)[nH]1</smiles>

Figure 1.

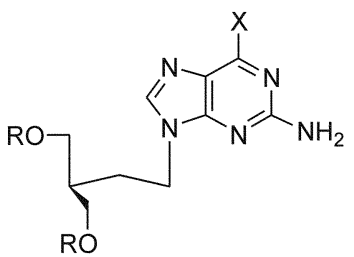

Penciclovir: $\mathrm{R}=\mathrm{H}, \mathrm{X}=\mathrm{OH}$ Famciclovir: $\mathrm{R}=\mathrm{Ac}, \mathrm{X}=\mathrm{H}$

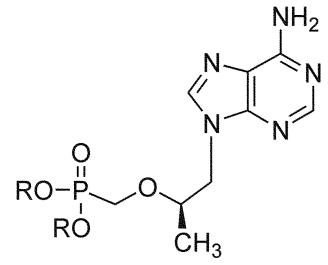

Bis(POC)PMPA: R =Isopropoxycarobonyloxylmethyl
"Corresponding author. Tel.: +82-62-230-6378; Fax: +82-62222-5414; c-nail: hongjh $\hat{a}$ mail.chosun.ac.kr for less toxic and more effective antiviral agents, which do not have any cross-resistance with existing drugs. In view of the stimulating results reported for acyclic nucleosides and as a part of our ongoing drug discovery efforts, this study aimed to synthesize novel 1',2'-seco-4'-C'-branched nucleosides. This paper reports their synthetic routes from the simple acyclic ketone derivatives $\mathbf{1 - 3}$.

\section{Results and Discussions}

As shown in Scheme 1, the synthetic route is straightforward. It was envisaged that a [3,3]-sigmatropic rearrangement of compounds 7-9 would produce the desired quatemary carbon compounds 10-12 with the required functional groups. Subjecting compounds 16-18 to nucleophilic substitution conditions and desilyation gave the desired nucleosides 2224.

Silyl protection of the hydroxyl group of the commercially available starting materials $\mathbf{1 - 3}$, followed by a HornerWadsworth-Emmons (HWE) reaction, ${ }^{9}$ provided the unsaturated ethyl ester 4 and the cisitrans isomeric mixtures of compounds 5, 6. It was not necessary to separate the isomers of compounds $\mathbf{5}$ and $\mathbf{6}$, as they merged into one isomer in the subsequent reactions.

The $\alpha \beta$-unsaturated ethyl esters 4-6 were reduced by DIBALH at $-20^{\circ}$ ' $\mathrm{C}$ in $\mathrm{CH}_{2} \mathrm{Cl}_{2}$ to give the allylic alcohols $7-9$ in an $83-97 \%$ yield, which were subjected to a standard Johnson ortho ester Claisen rearrangement ${ }^{|0|}$ using triethyl orthoacetate to produce the $\gamma \delta$-unsaturated esters 10-12 in $81-86 \%$ yield. The slow addition of DIBALH to a solution of the esters $10-12$ in $\mathrm{CH}_{2} \mathrm{Cl}_{2}$ at $-20^{\circ} \mathrm{C}$ furnished desired alcohols 13-15 in an $89-91 \%$ yield. The hydroxyl group of compounds 13-15 were mesylated by treating them with methanesulfonyl chloride (MsCl) in an anhydrous $\mathrm{CH}_{2} \mathrm{Cl}_{2}$ to give the key intermediates 16-18, which were coupled with the nucleobase (adenine) under well-known standard conditions $\left(\mathrm{K}_{2} \mathrm{CO}_{3}, 18-\mathrm{C}-6, \mathrm{DMF}\right)^{\prime \prime}$ to give the acyclic adenine derivatives $\mathbf{1 9 - 2 1}$ in a $42-48 \%$ two step yield. Although a small amount of the $N^{7}$-isomer ${ }^{\prime 2}$ (less than $5 \%$ ) of the adenine base were present, they could be readily differentiated [UV $(\mathrm{MeOH}) \lambda_{\max } 279 \mathrm{~nm}$ ]. The removal of the $t$-butyldimethyl- 


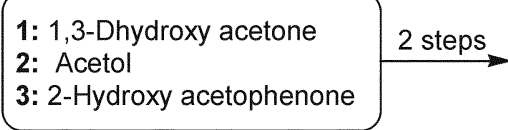<smiles>[R]C(C#CCOC)(CO)COC</smiles>

16: $\mathrm{R}=\mathrm{CH}_{2} \mathrm{OTBDMS}$

17: $\mathrm{R}=\mathrm{CH}_{3}$

18: $\mathrm{R}=\mathrm{C}_{6} \mathrm{H}_{5}$<smiles>[R]C(C#CCn1cnc2c(N)ncnc21)(COC)CO[Z17]([H])([H])C</smiles>

19: $\mathrm{R}=\mathrm{CH}_{2}$ OTBDMS

20: $\mathrm{R}=\mathrm{CH}_{3}$

21: $\mathrm{R}=\mathrm{C}_{6} \mathrm{H}_{5}$
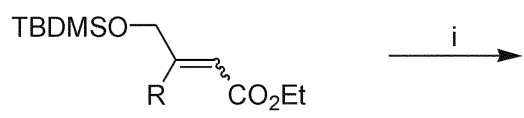

4: $\mathrm{R}=\mathrm{CH}_{2} \mathrm{OTBDMS}$

5: $\mathrm{R}=\mathrm{CH}_{3}$

6: $\mathrm{R}=\mathrm{C}_{6} \mathrm{H}_{5}$
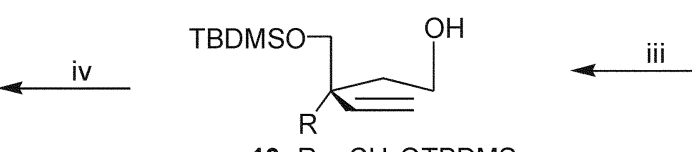

13: $\mathrm{R}=\mathrm{CH}_{2}$ OTBDMS

14: $\mathrm{R}=\mathrm{CH}_{3}$

15: $\mathrm{R}=\mathrm{C}_{6} \mathrm{H}_{5}$

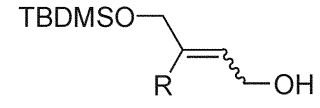

7: $\mathrm{R}=\mathrm{CH}_{2} \mathrm{OTBDMS}$

8: $\mathrm{R}=\mathrm{CH}_{3}$

9: $\mathrm{R}=\mathrm{C}_{6} \mathrm{H}_{5}$

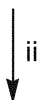

10: $\mathrm{R}=\mathrm{CH}_{2} \mathrm{OTBDMS}$

11: $\mathrm{R}=\mathrm{CH}_{3}$

12: $\mathrm{R}=\mathrm{C}_{6} \mathrm{H}_{5}$

Scheme I. Reagents: i) DIBAL.H. $\left(\mathrm{H}_{2} \mathrm{Cl}_{2} .-20{ }^{\circ} \mathrm{C} .2\right.$ h. 83-97\%: ii) Triethyl orthoacetate, Propionic acid. overnight. 130 " $\mathrm{C} .81-86 \%$ : iij)

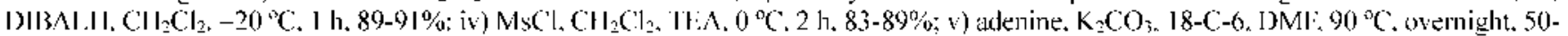
$54 \%$; vi) TBAF. THF rt. 3 h. $81-90 \%$.

Table 1. The antiviral activities of the sunthesized compounds

\begin{tabular}{|c|c|c|c|c|c|}
\hline & $\begin{array}{c}\text { IIIV-I } \\
\mathrm{EC}_{s i}(\mu \mathrm{g} / \mathrm{mL})\end{array}$ & $\begin{array}{c}\text { HISV-1 } \\
\mathrm{EC}_{S_{0}}(\mu \mathrm{g} / \mathrm{mL})\end{array}$ & $\begin{array}{c}\text { J1CMV } \\
\mathrm{EC}_{\mathrm{so}}(\mu \mathrm{g} / \mathrm{mL})\end{array}$ & $\begin{array}{c}C o x 133 \\
\mathrm{EC}_{50}(\mu \mathrm{g} / \mathrm{mL})\end{array}$ & $\begin{array}{c}\text { cytotoxicity } \\
I_{50}(\mu g / m L)\end{array}$ \\
\hline 22 & 10.40 & $>100$ & 62.16 & $>100$ & $>100$ \\
\hline 23 & $>100$ & $>100$ & $>100$ & 53.49 & $>100$ \\
\hline 24 & $>100$ & $>100$ & $>100$ & $>100$ & $>100$ \\
\hline$A / \mathrm{I}$ & 0.0005 & $N D$ & $\mathrm{NI}$ & $\mathrm{ND}$ & 0.5 \\
\hline Ganciclovir & NI) & 1.21 & VI) & $\mathrm{VI}$ & $>10$ \\
\hline Ribavirin & NI) & $\mathrm{NIJ}$ & VI) & 30.96 & $>300$ \\
\hline
\end{tabular}

ND: Nol Determined.

silyl (TBDMS) group of compounds 19-21 was accomplished by tetrabutylammonium fluoride (TBAF) to give the final nucleosides 22-24 in an $81-90 \%$ yield.

Antiviral assays of the synthesized nucleosides 22-24 against the human immunodeficiency virus 1 (HIV-I), the herpes simplex virus I (HSV-I), human cytomegalovirus (HCMV) and CoxB3 virus were performed. Although the tested compounds did not display any exceptional antiviral activity, the hydroxymethyl branched nucleoside $\mathbf{2 2}$ exhibited moderate anti-HIV activity in MT-4 cells (EC $\mathrm{EC}_{50}-$ $10.4 \mu \mathrm{mol})$ without any cytotoxicity up to $100 \mu \mathrm{mol}$ (Table 1). This result strongly suggests that this structure for the novel nucleosides might be a candidate for a new lead compound for the development of new antiviral agents.

\section{Conclusion}

Novel acyclic nucleosides were successfully synthesized starting from very simple ketone derivatives. This synthetic method is highly efficient and convenient, and can be further applied to the synthesis of geminally substituted novel acyclic nucleosides. Based on this strategy, the syntheses of the other 4'-modified acyclic nucleosides with different bases are in progress.

\section{Experimental Section}

The melting points were determined on a Mel-tem Il laboratory device and are uncorrected. The NMR spectra were recorded on a Bruker 300 Fourier transform spectro- 
meter. The chemical shifts are reported as parts per million $(\delta)$ and the signals are quoted as s (singlet). d (doublet). $\mathrm{t}$ (triplet). q (quartet). $\mathrm{m}$ (multiplet) and dd (doublet of doublets). The UV spectra were obtained using a Beckman DU-7 spectrophotometer. The elemental analyses were performed using an Elemental Analyzer System (Profile HV-3). TLC was performed on Uniplates (silica gel) purchased from Analtech $\mathrm{Co}$. All reactions were carried out under $\mathrm{N}_{2}$ unless otherwise specified. Dry dichloromethane. benzene and pyridine were obtained by distillation from $\mathrm{CaH}_{2}$. The dry THF was obtained by distillation from $\mathrm{Na}$ and benzophenone immediately prior to use.

4,4-Bis(t-butyldimethylsilyoxy)-iso-pent-2-en-1-ol (7): To a solution of 4 (10 g. $51.45 \mathrm{mmol})$ in $\mathrm{CH}_{2} \mathrm{Cl}_{2}(200 \mathrm{~mL})$. DIBALH (108 mL, 1.0 M solution in hexane) was added slowly at $-20^{\circ} \mathrm{C}$. and stirred for $2 \mathrm{~h}$ at $0^{\circ} \mathrm{C}$. To the mixture. methanol ( $108 \mathrm{~mL}$ ) was then added. The mixture was stirred at room temperature for $2 \mathrm{~h}$, and the resulting solid was filtered through a Celite pad. The filtrate was concentrated under vacuum. and the residue was purified by silica gel column chromatography (EtOAc/hexane. 1:4) to give the allỵlic alcohol 3 (16.78 g. 97\%) as a colorless oil: ' $\mathrm{H}$ NMR $\left(\mathrm{CDCl}_{3}, 300 \mathrm{MHz}\right) \delta 5.82$ (t. $\left.J=8 .+\mathrm{Hz}, \mathrm{lH}\right),+23$ (s. $\left.+\mathrm{H}\right)$. 4.16 (s. 2H). 0.92 (s. 18H) 0.00 (s. 12H).

$(E, Z)$-4-(t-Butyldimethylsilyloxymethyl)-3-methyl-but2-en-1-ol (8) and $(E, Z)-4$-(t-Butyldimethylsilyloxymethyl)3-phenyl-but-2-en-1-ol (9): Compound 8 and 9 were prepared from 5 and 6 , respectively as described for compound 7. Compound 8: yield 89\%: ${ }^{1} \mathrm{H} \mathrm{NMR}\left(\mathrm{CDCl}_{3}\right.$. $300 \mathrm{MHz}) \delta 5.68(\mathrm{br} \mathrm{s} . \mathrm{lH})+2 \mathrm{l}(\mathrm{d} J=6.6 \mathrm{~Hz} .2 \mathrm{H}) .4 .03(\mathrm{~s}$. $2 \mathrm{H}$ ) $1.77,1.64$ (s. s. $3 \mathrm{H}$ ). $0.9 \mathrm{l}$ (s. $9 \mathrm{H}), 0.07$ (s. $6 \mathrm{H}$ ): compound 9: yield 83\%: ${ }^{l} \mathrm{H}$ NMR $\left(\mathrm{CDCl}_{\hat{3}}, 300 \mathrm{MHz}\right) \delta$ $7.32-7.07$ (m.5H). 5.99.5.91 (dt. $J=6.6,6.6 \mathrm{~Hz}, \mathrm{IH}) .4 .31$ (d. $J=6.6 \mathrm{~Hz}, 1 \mathrm{H}), 4.27$ (s. IH). $0.85,0.81$ (s. s. 9H), 0.02 (m. 6H)

Ethyl 3,3-bis(t-butyldimethylsilyloxymethyl)-pent-4enoate (10): A solution of $15 \mathrm{~g}(+3.27 \mathrm{mmol})$ of allylic alcohol 7.150 $\mathrm{mL}$ of tiethyl orthoacetate. $0.78 \mathrm{~mL}$ of propionic acid was heated at $130-135{ }^{\circ} \mathrm{C}$ overnight with constant stirring under conditions for the removal of ethanol by distillation. The excess triethyl orthoaceate was distilled off and the residue was purified by silica gel column chromatography (EtOAc/hexane. $1: 15$ ) to give compound $10(15.5 \mathrm{~g} .86 \%)$ as a colorless oil: ${ }^{\mathrm{l}} \mathrm{H} \mathrm{NMR}\left(\mathrm{CDCl}_{3}, 300\right.$ $\mathrm{MHz}) \delta 5.87$ (dd. $J=18.0,11.4 \mathrm{~Hz}, 1 \mathrm{H}), 5.09$ (d. $J=11.1$ $\mathrm{Hz}, 1 \mathrm{H}),+.98(\mathrm{~d}, J=19.5 \mathrm{~Hz}, 1 \mathrm{H}), 4.05(\mathrm{q}, J=7.5 \mathrm{~Hz}, 2 \mathrm{H})$. 3.64 (dd $J=15.6 .9 .0 \mathrm{~Hz}) .2 .40$ (s. $2 \mathrm{H}) .1 .22$ (t. $J=7.5 \mathrm{~Hz}$. $3 \mathrm{H}), 0.85$ (s. $18 \mathrm{H}) .0 .01$ (s. $12 \mathrm{H}):{ }^{13} \mathrm{C} \mathrm{NMR}\left(\mathrm{CDCl}_{\mathfrak{j}}\right) \delta$ $1171.92,139.76,114.48,64.67,59.88,45.98,36.84,25.85$. 18.25. 14.25. $-5.56:$ Anal calc for $\mathrm{C}_{21} \mathrm{H}_{44} \mathrm{O}_{4} \mathrm{Si}_{2}:$ C. $60.52: \mathrm{H}$. 10.64. Found: C. $60.39:$ H. 10.87

( \pm )-3-(t-Butyldimethylsilyloxymethyl)-3-methyl-pent- - enoic acid ethyl ester (11) and ( \pm )-3-(t-Butyldimethylsilyloxymethyl)-3-phenyl-pent-4-enoic acid ethyl ester (12): Compound 11 and 12 were prepared from compounds 8 and 9. respectively as described for compound 10 . Compound 11: yield $83 \%$ : ${ }^{1} \mathrm{H}$ NMR $\left(\mathrm{CDCl}_{3} .300 \mathrm{MHz}\right) \delta 5.91$ (d. $J=$
$10.8 \mathrm{~Hz}, \mathrm{lH}), 5.89(\mathrm{~d}, J=11.4 \mathrm{~Hz}, \mathrm{IH}) .5 .05(\mathrm{~d}, J=1.2 \mathrm{~Hz}$. IH). 5.02 (d. $J=7.5 \mathrm{~Hz}, \mathrm{lH}) .4 .01$ (q. $J=7.2 \mathrm{~Hz}, 2 \mathrm{H}$ ). 3.46 (d. $J=9.3 \mathrm{~Hz}, \mathrm{lH}) .3 .4 \mathrm{l}(\mathrm{d}, J=9.3 \mathrm{~Hz}, \mathrm{lH}) .2 .40(\mathrm{~d}, J=3.3$ Hz. 2H), $1.23(\mathrm{t}, J=7.5 \mathrm{~Hz}, 3 \mathrm{H}), 1.2 \mathrm{l}(\mathrm{s}, 3 \mathrm{H}), 1.11$ (s. $9 \mathrm{H})$, 0.05 (s. $6 \mathrm{H}):{ }^{13} \mathrm{C} \mathrm{NMR}\left(\mathrm{CDCl}_{3}\right) \delta 171.94,143.11 .112 .99$. $69.91,59.83,41.39 .29 .25,25.66,20.70,14.26,-5.58$ : Anal calc for $\mathrm{C}_{15} \mathrm{H}_{3} \mathrm{O}_{3} \mathrm{Si}: \mathrm{C} .62 .89: \mathrm{H}, 10.55$. Found: C. $62.60: \mathrm{H}$. 10.45

Compound 12: yield 81\%: ${ }^{\mathrm{H}} \mathrm{NMR}\left(\mathrm{CDCl}_{2}, 300 \mathrm{MHz}\right) \delta$ $7.36-7.25(\mathrm{~m} .5 \mathrm{H}) .6 .26$ (dd. $J=18.0 .11 .1 \mathrm{~Hz}, \mathrm{lH}) .5 .31$ (dd. $J=11.4 .1 .2 \mathrm{~Hz}, \mathrm{IH}) .5 .16(\mathrm{dd} . J=17.7,0.6 \mathrm{~Hz}, \mathrm{lH}$ ). $4.10-3.99(\mathrm{~m} .4 \mathrm{H}), 3.00(\mathrm{~s} .2 \mathrm{H}) .1 .18$ (t. $J=6.9 \mathrm{~Hz}, 3 \mathrm{H})$. 0.99 (s. 9H), 0.02 (d. $J=8.1 .6 \mathrm{H}):{ }^{13} \mathrm{C} \mathrm{NMR}\left(\mathrm{CDCl}_{3}\right) \delta$ $171.51,143.17,142.33 .127 .82,127.34,126.30 .114 .34$. $67.73 .59 .94,48.70 .39 .74,25.76,18.19 .14 .07,-5.71:$ Anal calc for $\mathrm{C}_{2 \mathrm{i}} \mathrm{H}_{3} \mathrm{O}_{3} \mathrm{Si}:$ C. $68.92: \mathrm{H}, 9.25$. Found: C. 68.69: $\mathrm{H}$. 9.05 .

3,3-Bis( $t$-butyldimethylsilyloxymethyl)-4-penten-1-ol (13): To a solution of compound $10(5 \mathrm{~g} .11 .99 \mathrm{mmol})$ in $\mathrm{CH}_{2} \mathrm{Cl}_{2}(100 \mathrm{~mL})$. DIBALH $(25.2 \mathrm{~mL}, 1.0 \mathrm{M}$ solution in hexane) was added slowly at $-20^{\circ} \mathrm{C}$. and stirred for $\mathrm{l} h$ at 0 ${ }^{\circ} \mathrm{C}$. To the mixture. methanol $(25.2 \mathrm{~mL})$ was then added. The mixture was stirred at room temperature for $\mathrm{l} h$, and the resulting solid was filtered through a Celite pad. The filtrate was concentrated under vacuum and the residue was purified by silica gel column chromatography (EtOAc/hexane. $1: 5$ ) to give the alcohol $5(4.09 \mathrm{~g}, 91 \%)$ as a colorless oil: ${ }^{\mathrm{l}} \mathrm{H}$

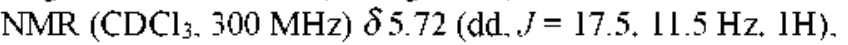
$5.15(\mathrm{~d}, J=11.0 \mathrm{~Hz}, \mathrm{lH}) .5 .12(\mathrm{~d} . J=18.0 \mathrm{~Hz}, \mathrm{lH}) .3 .98(\mathrm{t}$. $J=7.0 \mathrm{~Hz}, 2 \mathrm{H}) .3 .6 \mathrm{l}(\mathrm{d} . J=9.0 \mathrm{~Hz}, 4 \mathrm{H}) .1 .83(\mathrm{t} . J=8.1 \mathrm{~Hz}$, $2 \mathrm{H}, \mathrm{H}-2), 0.88$ (s. 18H), 0.01 (s. $12 \mathrm{H}):{ }^{13} \mathrm{C} \mathrm{NMR}\left(\mathrm{CDCl}_{3}\right) \delta$ $149.28,109.21,69.78,58.78,37.78,33.45,20.19,15.07$. -5.72: Anal calc for $\mathrm{C}_{19} \mathrm{H}_{42} \mathrm{O}_{3} \mathrm{Si}_{2}:$ C. $60.90: \mathrm{H}, 11.30$. Found: C. $60.67:$ H. 10.95

( \pm )-3-(t-Butyldimethylsilyloxymethyl)-3-methyl-pent4-en-1-0l (14) and ( \pm )-3-( $t$-Butyldimethylsilyloxymethyl)3-phenyl-pent-4-en-1-ol (15): Compound 14 and 15 were prepared from compounds 11 and $\mathbf{1 2}$, respectively as described for compound 13. Compound 14 : yield $90 \%:{ }^{\text {l}} \mathrm{H}$ $\mathrm{NMR}\left(\mathrm{CDCl}_{3}, 300 \mathrm{MHz}\right) \delta 5.89$ (dd, $\left.J=17.7 .11 .4 \mathrm{~Hz}, \mathrm{lH}\right)$. 5.06 (dd. $J=4.5,1.2 \mathrm{~Hz}, \mathrm{IH}) .5 .02$ (dd. $J=10.5,0.9 \mathrm{~Hz}$. IH). $3.67(\mathrm{dd} . J=11.7 .6 .3 \mathrm{~Hz}, \mathrm{lH}), 3.46(\mathrm{~d}, J=9.6 \mathrm{~Hz}$. lH). $3.38(\mathrm{~d}, J=9.6 \mathrm{~Hz}, \mathrm{HH}), 1.69(\mathrm{t}, J=5.7 \mathrm{~Hz}, 2 \mathrm{H}), 1.00$ (s. $3 \mathrm{H}) .0 .90$ (s. 9H), $0.06(\mathrm{~s}, 6 \mathrm{H}):{ }^{13} \mathrm{C} \mathrm{NMR}\left(\mathrm{CDCl}_{3}\right) \delta$ $144.45,112.80,70.81,59.34,41.18,25.84,21.04,18.29$. -5.52: Anal calc for $\mathrm{C}_{12} \mathrm{H}_{2} \mathrm{O} \mathrm{O}_{2} \mathrm{Si}: \mathrm{C} .63 .87: \mathrm{H}, 11.55$. Found C. $63.74: H, 11.61$ : compound 15: yield $89 \%$ : ${ }^{l} \mathrm{H}$ NMR $\left(\mathrm{CDCl}_{3} .300 \mathrm{MHz}\right) \delta 7.33-7.28(\mathrm{~m} .5 \mathrm{H}) .5 .82$ (dd. $J=17.4$. $10.8 \mathrm{~Hz}, \mathrm{IH}) .5 .33(\mathrm{~d} . J=10.7 \mathrm{~Hz}, \mathrm{IH}) .5 .13(\mathrm{~d} . J=10.5 \mathrm{~Hz}$ lH). 4.24 (t. $J=6.9 \mathrm{~Hz} .2 \mathrm{H}) .3 .80(\mathrm{~s} .2 \mathrm{H}), 1.72(\mathrm{~m} .2 \mathrm{H}), 0.90$ (s. 9H), $-0.02(\mathrm{~s} .6 \mathrm{H}):{ }^{13} \mathrm{C} \mathrm{NMR}\left(\mathrm{CDCl}_{3}\right) \delta 1+2.48,1+1.65$. $128.20,127.32,126.60,115.09 .68 .80,67.72,48.59,34.18$. 25.74. 18.13. -5.71: Anal calc for $\mathrm{C}_{18} \mathrm{H}_{3 i j} \mathrm{O}_{2} \mathrm{Si}: \mathrm{C} .70 .53: \mathrm{H}$. 9.87. Found: C. 70.26: H, 9.66.

1-O-Mesyl-3,3-bis(t-butyldimethylsilyloxymethyl)-tpentene (16): To a solution of the alcohol 13 (350 mg. 0.93 mmol) in anhydrous $\mathrm{CH}_{2} \mathrm{Cl}_{2}$, anhy'drous triethylamine $(0.24$ 
$\mathrm{mL})$ and $\mathrm{MsCl}(130 \mathrm{mg} .1 .12 \mathrm{mmol})$ was added at $0{ }^{\circ} \mathrm{C}$. The mixture was stirred at the same temperature for $1 \mathrm{~h}$. and quenched by a cold saturated $\mathrm{NaHCO}_{3}$ solution $(0.5 \mathrm{~mL})$. The mixture was extracted with $\mathrm{CH}_{2} \mathrm{Cl}_{2}(20 \mathrm{~mL})$ and water $(20 \mathrm{~mL})$. The organic layer was dried over anlydrous magnesium sulfate and filtered. The filtrate was concentrated under vacuum, and the residue (376 mg. 89\%) was then subjected to the subsequent coupling reaction without further purification. In order to obtain spectroscopic data. a small amount of sample was purified by flash silica gel column chromatography (EtOAc/hexane, 1:3): ${ }^{1} \mathrm{H}$ NMR $\left(\mathrm{CDCl}_{3}, 300 \mathrm{MHz}\right) \delta 5.77$ (dd. $\left.J=18.0,11.7 \mathrm{~Hz}, \mathrm{IH}\right), 5.17$ (d. $J=10.5 \mathrm{~Hz}) .5 .06($ d. $J=18.0 \mathrm{~Hz}, 1 \mathrm{H}) .4 .30$ (t. $J=7.5$ Hz, 2H). 3.56 (d. $J=9.0 \mathrm{~Hz}, 4 \mathrm{H}$ ). 2.97 (s. $3 \mathrm{H}$ ). 1.90 (t. $J=$ $8.1 \mathrm{~Hz}, 2 \mathrm{H}) .0 .89$ (s. $18 \mathrm{H}), 0.04$ (s. $12 \mathrm{H}$ ).

$( \pm)-1-O$-Methanesulfonic acid-3-( $t$-butyldimethylsilyloxymethyl)-3-methyl-pent-4-enyl ester (17) and ( \pm )-1- $O$ Methanesulfonic acid-3-( $t$-butyldimethylsilyloxymethyl)3-phenyl-pent-4-enyl ester (18): Compound 17 and 18 were prepared from compounds 14 and 15 . respectively as described for compounds 16 . Compound 17: yield $88 \%$ : ${ }^{1} \mathrm{H}$ NMR $\left(\mathrm{CDCl}_{\hat{j}}, 300 \mathrm{MHz}\right) \delta 5.82(\mathrm{~d}, J=10.8 \mathrm{~Hz}, \mathrm{lH}), 5.76$ (d. $J=11.1 \mathrm{~Hz} .1 \mathrm{H}$ ). 5.11 (dd. $J=10.8 .0 .9 \mathrm{~Hz}, \mathrm{IH}$ ). 5.00 (dd. $J=17.7,0.9 \mathrm{~Hz}, \mathrm{IH}), 4.25(\mathrm{t} . J=7.8 \mathrm{~Hz}, 2 \mathrm{H}), 3.36$ (dd. $J=18.9 .9 .3 \mathrm{~Hz}, 2 \mathrm{H}$ ). $2.98(\mathrm{~s} .3 \mathrm{H}), 1.91-1.85$ (m. $2 \mathrm{H}) .1 .02$ (s. $3 \mathrm{H}$ ). 0.90 (s. $9 \mathrm{H}$ ). 0.04 (s. $6 \mathrm{H}$ ): compound 18: yield $83 \%$ : ${ }^{l} \mathrm{H}$ NMR $\left(\mathrm{CDCl}_{\hat{\jmath}+} 300 \mathrm{MHz}\right) \delta 7.35-7.29(\mathrm{~m} .5 \mathrm{H}) .6 .02(\mathrm{dd}$. $J=17.4 .10 .8 \mathrm{~Hz}, \mathrm{IH}) .5 .34(\mathrm{~d} . J=9.9 \mathrm{~Hz}, 1 \mathrm{H}) .5 .17(\mathrm{~d} . J=$ $17.7 \mathrm{~Hz}, \mathrm{IH}$ ). +.25 (t. $J=6.9 \mathrm{~Hz}, 2 \mathrm{H}$ ). 3.83 (s. $2 \mathrm{H}$ ). 2.93 (s. $3 \mathrm{H}) .2 .44(\mathrm{~m} .2 \mathrm{H}) .0 .95(\mathrm{~s} .9 \mathrm{H}),-0.02(\mathrm{~s} .6 \mathrm{H})$.

9-[3,3-Bis(t-butyldimethylsilyoxymethyl)-4-penten-1-y]] adenine (19): A solution of the mesylate 16 (376 mg. 0.83 mmol). $\mathrm{K}_{2} \mathrm{CO}_{3}$ (231 mg. $1.67 \mathrm{mmol}$ ). 18-crown-6 (329 mg. $1.26 \mathrm{mmol}$ ). and adenine ( $113.5 \mathrm{mg} .0 .8+\mathrm{mmol})$ in dry DMF $(6.7 \mathrm{~mL})$ was stirred overnight at $90^{\circ} \mathrm{C}$. The mixture was cooled to room temperature and concentrated under vacuum. The residue was diluted with brine $(20 \mathrm{~mL})$ and extracted with $\mathrm{CH}_{2} \mathrm{Cl}_{2}(10 \mathrm{~mL} \times 4)$. The combined organic layer was dried over anhy̛drous $\mathrm{MgSO}_{4}$. filtered and concentrated under reduced pressure. The residue was purified by silica gel column chromatography $(\mathrm{MeOH} /$ $\left.\mathrm{CH}_{2} \mathrm{Cl}_{2}, \mathrm{I}: 10\right)$ to give compound $19(220 \mathrm{mg}, 54 \%)$ as a white solid: mp 168-170 ${ }^{\circ} \mathrm{C}$ : ${ }^{l} \mathrm{H}$ NMR $\left(\mathrm{CDCl}_{\hat{j} .} 300 \mathrm{MHz}\right) \delta$ 8.31 (s. IH). 7.71 (s. $1 \mathrm{H}), 5.76$ (dd. $J=18.0 .11 .1 \mathrm{~Hz} . \mathrm{IH}$ ). 5.61 (br s. $2 \mathrm{H}), 5.17$ (d. $J=11.1 \mathrm{~Hz}, 1 \mathrm{H}), 5.10(\mathrm{~d}, J=18.0$ $\mathrm{Hz}, 1 \mathrm{H}), 4.17(\mathrm{t}, J=8 .+\mathrm{Hz}, 2 \mathrm{H}), 3.56(\mathrm{dd}, J=13.2 .9 .3 \mathrm{~Hz}$. $4 \mathrm{H}) .1 .97(\mathrm{dd} . J=17.1,8.7 \mathrm{~Hz}, 2 \mathrm{H}), 0.84(\mathrm{~s}, 18 \mathrm{H}), 0.02(\mathrm{~s}$. $12 \mathrm{H}):{ }^{13} \mathrm{C} \mathrm{NMR}\left(\mathrm{CDCl}_{\mathfrak{j}}\right) \delta 155.28,152.88,140.29,139.78$. 115.24. 64.84. 45.90, 39.93, 32.44, 25.85, 18.22, -5.52: Anal calc for $\mathrm{C}_{24} \mathrm{H}_{45} \mathrm{~N}_{5} \mathrm{O}_{2} \mathrm{Si}_{2}:$ C. $58.61: \mathrm{H}, 9.22: \mathrm{N}, 14.24$. Found: C. 58.62: H. 9.16: N. 14.36.

(土)-9-[3-( $t$-Butyldimethylsilyloxymethyl)-3-methy]-tpent-1-enyl] adenine (20) and ( \pm )-9-[3-( $t$-Butyldimethylsilyloxymethyl)-3-phenyl-4-pent-1-enyl] adenine (21): Compound 20 and 21 were prepared from compounds 17 and 18. respectively as described for compound 19 . Compound 20: yield 52\%: mp 160-161 ${ }^{\circ} \mathrm{C}$ : ${ }^{\mathrm{l}} \mathrm{H}$ NMR
$\left(\mathrm{CDCl}_{3} .300 \mathrm{MHz}\right) \delta 8.33$ (s. lH). 7.74 (s. lH). 5.87 (br s. $2 \mathrm{H}) .5 .83$ (d. $J=10.8 \mathrm{~Hz}, \mathrm{lH}) .5 .77$ (d. $J=10.8 \mathrm{~Hz} . \mathrm{lH}$ ). $5.10($ dd $J=7.8,1.2 \mathrm{~Hz}, 1 \mathrm{H}), 5.06($ dd $J=14.1,0.9 \mathrm{~Hz}$. IH). 4.14 (t. $J=6.3 \mathrm{~Hz}, 2 \mathrm{H}) .3 .42$ (d. $J=10.2 \mathrm{~Hz}, \mathrm{IH}) .3 .35$ (d. $J=10.2 \mathrm{~Hz}, 1 \mathrm{H}), 1.97$ (t. $J=7.2 \mathrm{~Hz}, 2 \mathrm{H}) .1 .04$ (s. $3 \mathrm{H}$ ), 0.85 (s, 9H), -0.03 (s. $6 \mathrm{H}$ ): ${ }^{13} \mathrm{C}$ NMR $\left(\mathrm{CDCl}_{3}\right) \delta 155.43$. $152.88,150.07,142.71$. 140.24, 119.51, 70.48, 41.24, 40.34 36.97, 25.84, 20.25, 18.24, -5.54: Anal calc for $\mathrm{C}_{18} \mathrm{H}_{31} \mathrm{~N}_{5} \mathrm{OS}$ : C. $59.79:$ H. 8.64: N. 19.37. Found: C. $59.58:$ H. 8.72: N. 19.53: compound 21 : yield 50\%: mp 167-170 ${ }^{\circ} \mathrm{C}:{ }^{1} \mathrm{H}$ NMR $\left(\mathrm{CDCl}_{3} .300 \mathrm{MHz}\right) \delta 8.31$ (s. IH). $7.68(\mathrm{~s}, \mathrm{lH}), 7.42-7.26$ (m, 5H), 6.67 (br s. 2H), 6.13 (dd $J=17.7,11.1 \mathrm{~Hz}, \mathrm{lH}$ ) 5.39 (dd $J=10.8,0.6 \mathrm{~Hz} . \mathrm{lH}) .5 .27$ (d. $J=18.0 \mathrm{~Hz}, \mathrm{lH}$ ). 4.19 (t. $J=8.1 \mathrm{~Hz} .2 \mathrm{H}$ ). 3.90 (dd. $J=14.1 .9 .9 \mathrm{~Hz}, 2 \mathrm{H}$ ), $2.57-2.48$ (m. 2H). 0.87 (s. 9H), -0.03 (s. 6H): ${ }^{13} \mathrm{C}$ NMR $\left(\mathrm{CDCl}_{3}\right) \delta 155.44 .152 .20,149.75,142.51,1+1.64 .140 .09$. $128.25,127.42,126.67,115.42 .68 .70 .49 .02,40.73,25.76$. 18. 15, -5.70: Anal calc for $\mathrm{C}_{23} \mathrm{H}_{32} \mathrm{~N}_{5} \mathrm{OSi}: \mathrm{C}, 65.2 \mathrm{I}: \mathrm{H}, 7.85$ : N. 16.53. Found: C. 65.44: H. 7.69: N, 16.32.

9-[3,3-Bis(hydroxymethyl)-4-penten-1-yl] adenine (22): To a solution of compound 19 (100 $\mathrm{mg} .0 .2 \mathrm{mmol}$ ) in tetrahydrofuran $(3 \mathrm{~mL})$. tetrabutylammonium fluoride (TBAF) $\left(0.6 \mathrm{~mL}, 1.0 \mathrm{M}\right.$ solution in THF) was added at $0^{\circ} \mathrm{C}$. The misture was stirred at room temperature for $3 \mathrm{~h}$, and concentrated under reduced pressure. The residue was purified by silica gel column chromatography $(\mathrm{MeOH} /$ $\mathrm{CH}_{2} \mathrm{Cl}_{2} . \mathrm{L}: 5$ ) to give compound $22(47.4 \mathrm{mg} .90 \%)$ as a white solid: mp $184-186^{\circ} \mathrm{C}$ : UV $(\mathrm{MeOH}) \lambda_{\max } 261.5 \mathrm{~nm}:{ }^{1} \mathrm{H}$ NMR (DMSO-ds, $300 \mathrm{MHz}) \delta 8.12$ (s. IH). 7.17 (br s. 2H). $5.80(\mathrm{dd}, J=18.3 .11 .4 \mathrm{~Hz}, \mathrm{lH}), 5.14(\mathrm{~d} . J=8.7 \mathrm{~Hz}, \mathrm{lH})$. 5.08 (s. IH) 4.58 (br s. $2 \mathrm{H}), 4.12(\mathrm{t} . J=8.4 \mathrm{~Hz}, 2 \mathrm{H}), 3.40$ (s. $4 \mathrm{H}), 1.87(\mathrm{t} . J=8.4 \mathrm{~Hz}, 2 \mathrm{H}){ }^{3} \mathrm{C} \mathrm{C}$ NMR (DMSO- $\left.d_{6}\right) \delta 155.92$. $152.02,149.39,140.83,118.72,114.77 .63 .87,45.39,32.39$. 23.19. 19.24. 13.55. 10.21: Anal calc for $\mathrm{C}_{1} \mathrm{H}_{17} \mathrm{~N}_{5} \mathrm{O}_{2}: \mathrm{C}$. 54.74: H, 6.5I: N. 26.60. Found: C. 54.80: H. 6.49: N. 26.78.

( \pm )-9-[3-( $t$-Hydroxymethyl)-3-phenyl-4-pent-1-enyl] adenine (23) and $( \pm)-9-[3-(t-H y d r o x y m e t h y l)-3-p h e n y l-4-$ pent-1-enyl] adenine (24): Compound 23 and 24 were prepared from compounds $\mathbf{2 0}$ and $\mathbf{2 1}$, respectively as described for compound 22. Compound 23 : y ield $81 \%$ : mp $180-182{ }^{\circ} \mathrm{C}$ : UV $\left(\mathrm{H}_{2} \mathrm{O}\right) \lambda_{\text {max }} 260.5 \mathrm{~nm}$ : ${ }^{3} \mathrm{H}$ NMR (DMSO- $d_{6}$. $300 \mathrm{MHz}$ ) $\delta 8.13$ (s. 2H). 7.14 (br s. $2 \mathrm{H}$ ). 5.86 (d. $J=11.4$. IH). $5.80(\mathrm{~d}, J=10.5 \mathrm{~Hz}, \mathrm{IH}) .5 .08(\mathrm{~d}, J=5.1 \mathrm{~Hz}, \mathrm{lH}) .5 .03$ (s. $1 \mathrm{H}$ ) 4.70 (br s. IH). $4.13-3.99$ (m. 2H). 3.27 (d. $J=5.1$ Hz. IH). 3.23 (d. $J=5.1 \mathrm{~Hz}, \mathrm{lH}), 1.89-1.80(\mathrm{~m}, 2 \mathrm{H}), 1.00$ (s. $3 \mathrm{H}):{ }^{3} \mathrm{C}$ NMR (DMSO-d6) $\delta$ 149.29, 147.10. 143.59 . $141.40,140.53 .118 .619,113.36,41.05,36.67,34.91,20.45$ : Anal calc for $\mathrm{C}_{12} \mathrm{H}_{17} \mathrm{~N}_{5} \mathrm{O}:$ : C. 58.28: H. 6.93: N. 28.32 . Found: C. 58.57: H. 6.60: N. 28.56: compound 24: yield

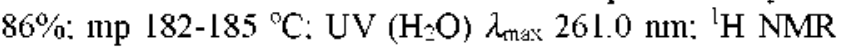
(DMSO-d6. $300 \mathrm{MHz}) \delta 8.12$ (s. 1H, H-8). 8.08 (s. lH). $7.41-7.15(\mathrm{~m}, 5 \mathrm{H}) .6 .05$ (dd. $J=15.9 .11 .1 \mathrm{~Hz}, 1 \mathrm{H}$ ). 5.27 (d. $J=11.7,1 \mathrm{H}), 5.17$ (s. $1 \mathrm{H}), 4.87$ (t. $J=6.4 \mathrm{~Hz}, 1 \mathrm{H}, \mathrm{D}_{2} \mathrm{O}$ exchangeable). $4.0 \mathrm{l}$ (br s. $2 \mathrm{H}$ ). $3.76(\mathrm{~m} .2 \mathrm{H}), 2.36$ (br d. $J=$ $7.8 \mathrm{~Hz}, 2 \mathrm{H}$ ): ${ }^{13} \mathrm{C}$ NMR (DMSO-d $\left.d_{6}\right) \delta 155.91,152.30,149.39$. $143.25,142.46,140.60,128.07,127.35,126.10,114.62$ $66.68,48.58,34.67,23.05,13.47$. Anal calc for $\mathrm{C}_{17} \mathrm{H}_{19} \mathrm{~N}_{5} \mathrm{O}$ 
C. $66.00:$ H. $6.19:$ N. 22.64. Found: C. $66.38:$ H. 6.31: N. 22.48 .

Acknowledgement. The authors wish to acknow ledge Dr. C.-K Lee (Korea Research Institute of Chemical Technology') for the antiviral assays.

\section{References}

1. Schaeffer. H. J: Beauchamp. L.: De Miranda, P: Elion, G .B: Bauer. D. J.: Collins. P. Nature 1978. 272. 583.

2. El Ashry. E. S. H.: El Kilany. Y. Acyclonucleosides: Part 1.2. 3. in Atrances in Heterocylic Chentistry: Academic Press: 1997: Vol. 67.68 .69 .

3. De Clercy. E.: Descmps. I.: De Somer. P: Holv. A. Sciente 1978. 200, 563. (b) Martin. I. C.: Dvorak C. A.: Smee. D. F.; Matthew; T. R.: Verheyden. J. P. H. J. Hed Chem 1983 26, 759. (c) Smith. K. O.: Galloway. K. S.: Kennell. W. L.: Oglivie. K. K.: Radatus. B. K. Antomicroh. Agents Chemother, 1982. 22. 55 (d) Vandendriessche. F.: Snoeck. R.: Janssen. G.: Hoogmartens. J.: Van Aerschot. A.: De Clereq. E.: Herdwijn. P. J. Med. Chem. 1992. 35. 1458.

4. Krenitsky, T. A.: Hall, W. W: De Miranda, P: Beauchamp. L. M.: Schaeffer. H. I.: Whiteman. P. D. Proc Katl Acad. Sci. LSA 1984.
81.3209 .

5. (a) Crumpacker. C. S. N. Engl J. Med. 1996. 335. 721. (b) Hamzeh. F. M.: Lietman. P. S. Antinterob. Agents Chemothet: 1991. 35. 1818 .

6. Earnshaw: D. L.: Bacon. T. H.; Darlison, S. J.; Edmonds, K.; Perkins, R. M: Vere Hodge, R. A. Antinticrob. Agents Chemother. 1992. 36, 2747.

7. Vere Hodge. R. A.: Sutton. D.: Boyd. M. R.: Harnden. M. R.: Jarvest. R. L. Animicob Agents Chemother. 1989. 33. 1765.

8. Arimilli. M. N.: Kim. C. U.: Dougherty. J.: Mulato. A.: Oliyai. R.: Shaw, J. P.: Cundy, K. C.: Bischofberger, N. Amiviral Chen. Chenother. $1997,8.557$.

9. Jeong. L. S.; Lee. Y. A.: Moon. H. R.; Chun, M. W. Nucleosides \& Nucleotides $1998.17,1473$

10. (a) Ko. O. H.: Hong. T. H. Tetahedron Lett. 2002. 43. 6399. (b) Hong. T. H.: Gao. M. Y.: Chu. C. K. Tetrohedron Lett. 1999. to. 231

11. Lee. J. Y: Oh. C. H.: Ko, O. H.: Hong, J. H. Nucleosides, Nucleotides \& Nuclaic Acids 2012, 21. 709. (b) Hossain. N. Rozenski. J.; De Clereq. E.: Herdewjn. P. Tetrahedron 1996, 52. 13655 .

12. (a) Panzica. R. P.: Rousseau. R. J.: Robins. R. K.: Townsend. L. B. J. Am. Chem. Soc. 1972. 94. 4708. (b) Rousseaul. R. J.: Robins. R. K.; Townsend, L. B. J. Ant. Chent. Soc. 1968,90.2661. 